\title{
IGF-1 reduces the apoptosis of endothelial progenitor cells induced by oxidized low-density lipoprotein by the suppressing caspasse-3 activity
}

\author{
Yonggang $\mathrm{Wu}^{1,2}$, Qiru Wang ${ }^{1}$, Lamei Cheng ${ }^{1}$, Jian Wang ${ }^{1}$, Xuan Sun ${ }^{1}$, Guangxiu Lu ${ }^{1}$ \\ ${ }^{1}$ Institute of Reproductive and Stem Cell Engineering, Central South University, National Center of human Stem Cell Research \\ and Engineering, Changsha, 410078 Hunan, China; ${ }^{2}$ Nuclear medicine Department, Second Xiangya Hospital, Central South \\ University, Changsha 410011, Hunan, China
}

Dyslipidemia increase the risk for coronary heart disease in part by impairing endothelial integrity. Endothelial progenitor cells(EPCs) play a pivotal role in reendotheliazation. In previous study, oxidized low-density lipoprotein (oxLDL) affects the apoptosis of the EPCs. However, the precise mechanism is still unclear. Therefore, we focused in this study to understand whether oxLDL can induce caspase-3 activity and insulin like growth factor-1(IGF-1) can prevent the EPC by the decrease the caspase-3 activity and the apoptosis in the EPCs. EPCs were isolated from peripheral blood and characterized. The EPCs were cultured with IGF-1 for $30 \mathrm{~min}$ and then were treated with oxLDL for $48 \mathrm{~h}$. The apoptosis of the Endothelial progenitor cells was checked by the flow cytometry and, while caspase-3 activity was measured simultaneously. After the EPC were treated, the mean of apoptotic rates and caspase-3 enzyme activity significantly decreased. in EPCs treated with IGF-1, the decrease of the caspase-3 activity by oxLDL was blocked and apoptosis prevented. Therefore, IGF-1 can reduced the apoptosis of endothelial progenitor cells induced by oxLDL. This effect may be mediated by significantly suppressing caspase- 3 activity.

Keywords: EPCs, oxLDL, IGF-1

Cell Research (2008) 18:s159. doi: 10.1038/cr.2008.249; published online 4 August 2008

Correspondence: Guangxiu Lu

E-mail: lugxdirector@yahoo.com.cn 